\title{
Bone health among patients with primary aldosteronism: a systematic review and meta-analysis
}

\author{
Huai H. LOH ${ }^{1}$ *, Anne YEE ${ }^{2}$, Huai S. LOH ${ }^{3}$
}

${ }^{1}$ Faculty of Medicine and Health Sciences, University of Malaysia Sarawak, Malaysia; ${ }^{2}$ Department of Psychological Medicine, Faculty of Medicine, University of Malaya, Malaysia; ${ }^{3}$ Clinical Academic Unit, Newcastle University Medicine Malaysia, Malaysia

*Corresponding author: Huai Heng Loh, Faculty of Medicine and Health Sciences, University of Malaysia Sarawak, Jalan Dato Muhammad Musa, 94300 Kota Samarahan, Sarawak, Malaysia. E-mail: hhlohdr@gmail.com

\section{A B S T R A C T}

INTRODUCTION: Recent studies showed a possible association between hyperaldosteronism and secondary hyperparathyroidism leading to reduced bone health, however results are conflicting.

EVIDENCE ACQUISITION: We conducted a meta-analysis to evaluate the relationship between primary aldosteronism (PA) with bone biochemical markers and to assess bone mineral density in patients with primary aldosteronism.

EVIDENCE SYNTHESIS: A total of 939 subjects were examined (37.5\% with PA). Patients with PA had significantly higher serum parathyroid hormone, lower serum calcium, higher urine calcium excretion and higher serum alkaline phosphatase compared to patients without PA, with no significant difference in serum vitamin D between both groups. Bone mineral density of lumbar spine, femoral neck and total neck of femur were similar between two groups. With PA treatment, there was a significant increment in serum calcium and reduction in serum parathyroid hormone.

CONCLUSIONS: PA is associated with hypercalciuria with subsequent secondary hyperparathyroidism. This potentially affects bone health. We recommend this to be part of complication screening among patients with PA.

(Cite this article as: Loh HH, Yee A, Loh HS. Bone health among patients with primary aldosteronism: a systematic review and meta-analysis. Minerva Endocrinol 2019;44:000-000. DOI: 10.23736/S0391-1977.18.02867-5)

KEY WORDS: Bone density; Calcium; Bone fractures; Hyperaldosteronism; Osteoporosis; Secondary hyperparathyroidism.

\section{Introduction}

$\mathrm{P}$ rimary aldosteronism (PA) is a disorder of the adrenal gland, causing autonomous production of aldosterone. It is the most common cause of secondary hypertension among middleaged adults. ${ }^{1-3}$ It is well-known to have detrimental effects on cardiovascular and renal system. ${ }^{4}$ Osteoporosis leading to bone fractures impairs quality of life and is associated with increased morbidity and mortality. ${ }^{5-7}$

Animal studies showed that rats which were given aldosterone and salt had a reduction in bone mineral density and cortical bone strength. ${ }^{8}$ This led to a few recent studies which have identified the possible association between hyperaldosteronism and reduced bone health and increased fracture risk. ${ }^{9-14}$

Although the exact mechanism is unknown, reduced bone health in patients with hyperaldosteronism was believed to be due to hypermagnesuria and hypercalciuria leading to bone loss. ${ }^{15}$ Recent studies also found an association between hyperaldosteronism and secondary hy- 